PROCEEDINGS OF THE

AMERICAN MATHEMATICAL SOCIETY

Volume 136, Number 1, January 2008, Pages 61-71

S 0002-9939(07)09025-9

Article electronically published on August 14, 2007

\title{
BOUNDS OF DIVIDED UNIVERSAL BERNOULLI NUMBERS AND UNIVERSAL KUMMER CONGRUENCES
}

\author{
ARNOLD ADELBERG, SHAOFANG HONG, AND WENLI REN
}

(Communicated by Wen-Ching Winnie Li)

\begin{abstract}
Let $p$ be a prime. We obtain good bounds for the $p$-adic sizes of the coefficients of the divided universal Bernoulli number $\frac{\hat{B}_{n}}{n}$ when $n$ is divisible by $p-1$. As an application, we give a simple proof of Clarke's 1989 universal von Staudt theorem. We also establish the universal Kummer congruences modulo $p$ for the divided universal Bernoulli numbers for the case $(p-1) \mid n$, which is a new result.
\end{abstract}

\section{INTRODUCTION}

There are many beautiful and useful congruences in number theory. Some examples are Wilson's theorem, Fermat's little theorem, Wolstenholme's theorem, Lucas' congruence, Kummer's congruences, and Glaisher's congruence, etc. By using Washington's p-adic expansion of certain reciprocal power sums of positive integers [13, Hong [7] obtained a generalization of Glaisher's congruence. Recently, Adelberg [3] gave a generalization of Wilson's theorem while Ren, Hong, and Zhou 12 generalized Adelberg's result and hence also extended Wilson's theorem. In this paper, our main interest is the universal Kummer congruences.

Let $c_{1}, c_{2}, \ldots$ be indeterminates over $\mathbf{Q}$ and let

$$
F(t)=t+c_{1} \frac{t^{2}}{2}+c_{2} \frac{t^{3}}{3}+\cdots .
$$

Let $G(t)=F^{-1}(t)$ be the compositional formal power series inverse of $F(t)$, namely $F(G(t))=G(F(t))=t$. The universal Bernoulli numbers $\hat{B}_{n}$ are defined by

$$
\frac{t}{G(t)}=\sum_{n=0}^{\infty} \hat{B}_{n} \frac{t^{n}}{n !}
$$

Evidently we have $\hat{B}_{n} \in \mathbf{Q}\left[c_{1}, c_{2}, \ldots, c_{n}\right]$. Actually $\hat{B}_{n}$ is a non-trivial $\mathbf{Q}$-linear combination of all the monomials of weight $n$, where $c_{i}$ has weight $i$. So $\hat{B}_{n}$ is the sum of $p(n)$ monomials, where $p(n)$ is the partition function.

Received by the editors July 5, 2006 and, in revised form, December 1, 2006.

2000 Mathematics Subject Classification. Primary 11B68, 11B83; Secondary 11A07.

Key words and phrases. Divided universal Bernoulli numbers, universal von Staudt theorem, universal Kummer congruence, $p$-adic valuation.

The second author is the corresponding author and was supported by New Century Excellent Talents in University Grant \# NCET-06-0785, and by SRF for ROCS, SEM. 
If we substitute $c_{i}=(-1)^{i}$, then $F(t)=\log (1+t)$ so that $G(t)=e^{t}-1$, and we obtain the classical Bernoulli numbers $B_{n}=\hat{B}_{n}$. The periodic behavior of the divided Bernoulli numbers $\frac{B_{n}}{n}$ is closely related to the existence of a $p$-adic zeta function (see [10]). The classical Kummer congruences concern the congruence relations among the divided Bernoulli numbers $\frac{B_{n}}{n}$. Specifically they state that if $p$ is a prime and $(p-1) \nmid n$ and $n \equiv m(\bmod p-1)$, then $\frac{B_{n}}{n} \equiv \frac{B_{m}}{m}(\bmod p)$. Clarke [4] showed that the divided universal Bernoulli number $\frac{\hat{B}_{n}}{n}$ is $p$-integral if $(p-1) \nmid n$ which is part of his universal von Staudt theorem. Adelberg [2] set up the universal Kummer congruences modulo $p$ for the divided universal Bernoulli numbers $\frac{\hat{B}_{n}}{n}$ when $(p-1) \nmid n$. Consequently Adelberg [3] obtained the universal Kummer congruences modulo powers of $p$ for the case $(p-1) \nmid n$. However the question of the universal Kummer congruences for the divided universal Bernoulli numbers when $(p-1) \mid n$ has not previously been answered.

In the present paper, we investigate the universal Kummer congruence modulo $p$ for the divided universal Bernoulli numbers for the remaining case $(p-1) \mid n$. We will first get good bounds for the $p$-adic valuations of the coefficients of the divided universal Bernoulli number $\frac{\hat{B}_{n}}{n}$ when $n$ is divisible by $p-1$. As an application, we give a simple proof to Clarke's 1989 universal von Staudt theorem [4] which generalized the theorems of Dibag [6], Ray [11, Katz [9], and Hurwitz [8]. Finally we establish new universal Kummer congruences modulo $p$ for the remaining case when $(p-1) \mid n$.

\section{Critical Bounds For the Divided universal Bernoulli numbers}

We follow the notations of $\left[\underline{1}-\underline{3}\right.$ throughout this paper. If $u=\left(u_{1}, u_{2}, \ldots\right) \in$ $\mathbf{N}^{\infty}$ with $u_{i}=0$ if $i \gg 0$ and $w(u):=\sum i u_{i}$, we identify $u$ with a partition of $w(u)$, where $u_{i}$ is the number of occurrences of the part $i$ in the partition. If $d(u):=\sum u_{i}$, then $d(u)$ is the number of parts in the partition. We call $w(u)$ the weight of $u$ and $d(u)$ the degree of $u$. If $u_{i}=0$ for $i>n$, we write $u \in \mathbf{N}^{n}$.

As usual, we let $v=v_{p}$ be the normalized $p$-adic valuation of $\mathbf{Q}$, i.e., $v(a)=b$ if $p^{b} \| a$. We can extend $v$ to $\mathbf{Q}\left[c_{1}, c_{2}, \ldots\right]$ by $v\left(\sum a_{u} c^{u}\right)=\min \left\{v\left(a_{u}\right)\right\}$ when $u=\left(u_{1}, \ldots, u_{n}\right) \in \mathbf{N}^{n}$ and $c^{u}=c_{1}^{u_{1}} \cdots c_{n}^{u_{n}}$. By the Lagrange inversion [1, 4], we have

$$
\frac{\hat{B}_{n}}{n}=\sum_{w=n} \tau_{u} c^{u}
$$

where $u=\left(u_{1}, \ldots, u_{n}\right) \in \mathbf{N}^{n}, w=w(u), d=d(u), c^{u}=c_{1}^{u_{1}} \cdots c_{n}^{u_{n}}, \gamma_{u}=2^{u_{1}} \ldots$ $(n+1)^{u_{n}} u_{1} ! \cdots u_{n}$ ! and

$$
\tau_{u}=\frac{(-1)^{d-1}(n+d-2) !}{\gamma_{u}} .
$$

The following facts are well known, and we will freely use them: For $x, y \in \mathbf{Z}^{+}$,

$$
v((x p) !)=x+v(x !), \quad v((x+y) !) \geq v(x !)+v(y !), \quad v(x !)=\frac{x-\sigma_{p}(x)}{p-1},
$$

where $\sigma_{p}(x)=\sum_{i=0}^{a} x_{i}$ is the base $p$ digit sum, if $x=\sum_{i=0}^{a} x_{i} p^{i}$, with digits $0 \leq x_{i} \leq p-1$.

We will use the explicit formula (2) for $\hat{B}_{n} / n$ in terms of the partitions of $n$ throughout this paper. The following theorem extends Proposition 3.2 of [3] by 
establishing $p$-adic estimates of the terms when $p$ is an odd prime and $(p-1) \mid n$. The proof is quite similar, differing only in consideration of the exceptional partitions that occur when $(p-1) \mid n$.

Theorem 2.1. Let $p$ be an odd prime and suppose $w(u)=n$ and $n=(p-1) s$ and $u_{p-1}<s$. Let $e=v\left(\gamma_{u}\right)-v\left(\left(p u_{p-1}\right) !\right)$ and $n^{\prime}=n-(p-1) u_{p-1}$. Then

$$
n+d-2 \geq\left(u_{p-1}+e+1\right) p
$$

except for the following cases where $\left(u_{p-1}+e+1\right) p>n+d-2 \geq\left(u_{p-1}+e\right) p$ :

(i) $p \geq 3, u_{p-1}=s-1$, and for $1 \leq i \leq \frac{p-3}{2}$, we have $u_{i}=u_{p-1-i}=1$, or if $i=\frac{p-1}{2}$, we have $u_{i}=2$. In these cases, $n^{\prime}=p-1$ and $e=0$.

(ii) $p=3, u_{2}=s-4, u_{8}=1$. In this case, $n^{\prime}=8$ and $e=2$.

Proof. Note that the hypotheses imply that $n \geq n^{\prime} \geq p-1$ since $(p-1) \mid n^{\prime}$. Let $u^{\prime}$ be the partition such that $u_{p-1}^{\prime}=0$ and $u_{i}^{\prime}=u_{i}$ if $i \neq p-1$. Then we have $w\left(u^{\prime}\right)=n^{\prime}$ and $v\left(\gamma_{u^{\prime}}\right)=e$. Let $d^{\prime}=d\left(u^{\prime}\right)$. Also $n+d-2=p u_{p-1}+n^{\prime}+d^{\prime}-2$, so replacing $u$ by $u^{\prime}$, we can assume that $u_{p-1}=0$.

Clearly we now have $n+d=\sum_{i=1}^{n}(i+1) u_{i}$ and $e=\sum_{i=1}^{n} e_{i}$, where

$$
e_{i}:=v(i+1) u_{i}+v\left(u_{i} !\right) \text {, }
$$

so $e_{i}>0$ only when $p \mid(i+1)$ and $u_{i}>0$ or $u_{i} \geq p$.

Let us consider the $\frac{p-1}{2}$ exceptional cases in (i), where $n^{\prime}=p-1$ and $d^{\prime}=2$. If $i<\frac{p-1}{2}$ and $j=p-1-i$, then $e_{i}=e_{j}=0$ and $(i+1) u_{i}-2 \leq(j+1) u_{j}-2<p$, while if $i=\frac{p-1}{2}$, then again $e_{i}=0$ and $(i+1) u_{i}-2=p-1<p$. For the exceptional case (ii) with $p=3, i=8, u_{i}=1, e_{i}=2$, we have $(i+1) u_{i}=9=\left(e_{i}+1\right) p$.

We assert that

$$
(i+1) u_{i} \geq\left(e_{i}+1\right) p+2
$$

for all cases where $e_{i}>0$ and $i \neq p-1$, apart from case (ii) and the exceptional cases $i=1, u_{i}=p$ and $i=2 p-1, u_{i}=1$, i.e.,

$$
(i+1) u_{i} \geq\left(v(i+1) u_{i}+v\left(u_{i} !\right)+1\right) p+2
$$

if $i \neq p-1$ and $p \mid(i+1)$ or $u_{i} \geq p$, apart from case (ii) and the exceptional cases $i=1, u_{i}=p$ and $i=2 p-1, u_{i}=1$. In all these cases we have $(i+1) u_{i}=\left(e_{i}+1\right) p$.

Since the right-hand side of the inequality (6) involves only $v(i+1)$ rather than $i+1$, it suffices to take $i+1$ minimal for given $v(i+1)$, i.e., we have only to consider the cases $i=1$ and $u_{i} \geq p, i=2 p-1$ and $u_{i} \geq 1$, and $i=p^{\alpha}-1$ with $\alpha \geq 2$ and $u_{i} \geq 1$. In all cases the inequality follows easily using the estimate $v\left(u_{i} !\right) \leq \frac{u_{i}-1}{p-1} \leq \frac{u_{i}-1}{2}$. The details follow.

First assume that $p \nmid(i+1)$ and $u_{i} \geq p$. If $i \geq 2$ and $u_{i}=p$, inequality (6) holds since $3 p \geq 2 p+2$. If $i=1$ and $p+1 \leq u_{i}<2 p$, then inequality (6) holds since $2(p+1)=2 p+2$. Finally let $i=1$ and $u_{i}=k p+f$ where $k \geq 2$ and $0 \leq f<p$. Then it will suffice to prove that $2(k p) \geq(k+v(k !)+1) p+2$, which is true since $v(k !) \leq(k-1) / 2$, so $k-1-v(k !) \geq 1$ if $k \geq 2$, and hence $p(k-1-v(k !)) \geq 2$. This concludes the case where $p \nmid(i+1)$.

Second assume that $\alpha=v(i+1) \geq 1$. If $\alpha=1$, then let $i+1=k p$. So (6) is equivalent to $k p u_{i} \geq\left(u_{i}+v\left(u_{i} !\right)+1\right) p+2$ if $k \geq 2$ and $u_{i} \geq 1$, except if $k=2$ and $u_{i}=1$. But this inequality is equivalent to $p\left((k-1) u_{i}-1-v\left(u_{i} !\right)\right) \geq 2$, which is true since $v\left(u_{i} !\right)<(k-1) u_{i}-1$ if $k \geq 2$ and $u_{i} \geq 1$ except if $k=2$ and $u_{i}=1$. 
Consequently, assume that $\alpha \geq 2$. As previously noted, it suffices to take $i=$ $p^{\alpha}-1$, and (6) is then equivalent to $p\left(\left(p^{\alpha-1}-\alpha\right) u_{i}-1-v\left(u_{i} !\right)\right) \geq 2$, which is true if $\alpha \geq 2$, and $u_{i} \geq 1$ with the single exception $\alpha=2, p=3$, and $u_{i}=1$. Since $p^{\alpha-1}-\alpha=1$ if $p=3$ and $\alpha=2$, and in all other cases $p^{\alpha-1}-\alpha>1$, so $v\left(u_{i} !\right)<\left(p^{\alpha-1}-\alpha\right) u_{i}-1$ and hence $(6)$ holds as specified.

Finally if $u_{i} \geq 1$, then $(i+1) u_{i} \geq 2$, while if $(i+1) u_{i} \geq\left(e_{i}+1\right) p$ and $(j+1) u_{j} \geq$ $\left(e_{j}+1\right) p$, then $(i+1) u_{i}+(j+1) u_{j} \geq\left(e_{i}+e_{j}+1\right) p+p \geq\left(e_{i}+e_{j}+1\right) p+2$. Since $u_{p-1}<s$, there exists an $i \neq p-1$ such that $u_{i} \geq 1$. If $e=0$, then as previously noted, since $n \geq p-1$, we have $n+d-2 \geq(e+1) p$ except $n=p-1, d=2$, which is case (i). Since $(p-1) \mid n^{\prime}$, the desired inequality of the theorem for $n+d-2$ now follows by adding the "local" inequalities for each part $i$ separately.

The proof of Theorem 2.1 is complete.

Theorem 2.2. Suppose $p$ is an odd prime and $w(u)=n$ and $n=s(p-1)$ and $u_{p-1}<s$. Then we have

(i) $v\left(\tau_{u}\right) \geq 1$ except for case (i) of Theorem 2.1 and except for case (ii) of Theorem 2.1 if $s \equiv 1(\bmod 3)$;

(ii) $v\left(\tau_{u}\right)=0$ in case (i) of Theorem 2.1 and case (ii) of Theorem 2.1 if $s \equiv 1$ $(\bmod 3)$.

Proof. With the same notation as in Theorem 2.1, if $n+d-2 \geq\left(u_{p-1}+e+1\right) p$, then $v((n+d-2) !) \geq v\left(\left(\left(u_{p-1}+e+1\right) p\right) !\right) \geq v\left(\left(p u_{p-1}\right) !\right)+e+1$. So $v\left(\tau_{u}\right) \geq 1$.

Now we consider the exceptions of Theorem 2.1: (i) If $p \geq 3, u_{p-1}=s-1$, and $d^{\prime}=2$, then $n+d-2=s p-1$, so $v((n+d-2) !)=v((s p-1) !)=v(((s-1) p) !)=$ $s-1+v((s-1) !)=v\left(\gamma_{u}\right)$ and $v\left(\tau_{u}\right)=0$.

For case (ii) with $p=3$, we have $d=s-3$ so $n+d-2=3 s-5$ and

$$
\begin{aligned}
v\left(\tau_{u}\right) & =v\left(\frac{(3 s-5) !}{(s-4) ! 3^{s-4} 3^{2}}\right) \\
& =v((s-2)(s-3))+v((3 s-5)(3 s-7)(3 s-8)(3 s-10)(3 s-11)),
\end{aligned}
$$

so $v\left(\tau_{u}\right)=0$ if $s \equiv 1(\bmod 3)$ and otherwise $v\left(\tau_{u}\right)>0$. Hence Theorem 2.2 is proved.

For any positive odd integer $a$, we define the double factorial $a$ !! of $a$ by $a ! !:=$ $\prod_{1 \leq k \leq a,(2, k)=1} k$. That is, $a ! !=a \cdot(a-2) \cdot \ldots \cdot 3 \cdot 1$.

Theorem 2.3. Let $p=2$ and $w(u)=n$. Then $v\left(\tau_{u}\right)>0$ apart from the following exceptions:

(i) If $u_{1}=n$, then $v\left(\tau_{u}\right)=-(1+v(n))$.

(ii) If $n \geq 3$ and $u_{1}=n-3, u_{3}=1$, then $v\left(\tau_{u}\right)=-1$ if $n$ is odd, and $v\left(\tau_{u}\right)=0$ if $4 \mid n$.

(iii) If $n \geq 6$ and $u_{1}=n-6, u_{3}=2$, then $v\left(\tau_{u}\right)=-1$ if $n \equiv 2(\bmod 4)$, and $v\left(\tau_{u}\right)=0$ if $8 \mid n$.

(iv) If $n \geq 12$ and $u_{1}=n-12, u_{3}=4$, then $v\left(\tau_{u}\right)=0$ if $n \equiv 4(\bmod 8)$.

(v) If $n \geq 2$ and $u_{1}=n-2, u_{2}=1$, then $v\left(\tau_{u}\right)=0$.

Proof. First note that $v\left(\left((i+1) u_{i}\right) !\right)=e_{i}$ holds for $i=1$, where $e_{i}$ is defined as in (4). Now let $i>1$ and $i+1=r 2^{\alpha}$ with $r$ odd. Then $e_{i}=\alpha u_{i}+v\left(u_{i} !\right)$. Using $v\left(\left(2^{\alpha} u_{i}\right) !\right)=\left(2^{\alpha}-1\right) u_{i}+v\left(u_{i} !\right)$, which can easily be proved by induction on $\alpha$, since $2^{\alpha}-1 \geq \alpha$, it follows easily that $v\left(\left((i+1) u_{i}\right) !\right) \geq e_{i}$, and if $r>1$ and $u_{i}>0$, then $v\left(\left((i+1) u_{i}-2\right) !\right) \geq e_{i}+1$, unless $i=2$ and $u_{i}=1$. 
We next show that apart from the exceptional cases involved in (i)-(v), if $u_{i}>0$, then we have

$$
v\left(\left((i+1) u_{i}-2\right) !\right) \geq e_{i}+1
$$

First consider the case $\alpha=0$, so $i+1=r \geq 3$. Then $r u_{i}-2 \geq u_{i}+2$ unless $r=3, u_{i}=1$, whence $v\left(\left(r u_{i}-2\right) !\right) \geq v\left(u_{i} !\right)+1=e_{i}+1$ apart from exceptional case (v), verifying (7) in this case.

Next assume $\alpha>0$. By the above result we may let $r=1$. Then $v\left(\left(2^{\alpha} u_{i}-2\right) !\right)=$ $2^{\alpha-1} u_{i}-1+v\left(\left(2^{\alpha-1} u_{i}-1\right) !\right)$. If $\alpha>3$, then $2^{\alpha-1}>\alpha+1$ and $2^{\alpha-1} u_{i}-1 \geq 2$. Thus $v\left(\left(2^{\alpha} u_{i}-2\right) !\right)>(\alpha+1) u_{i} \geq e_{i}+1$ since $u_{i} \geq v\left(u_{i} !\right)+1$. If $\alpha=3$, then $v\left(\left(2^{\alpha} u_{i}-2\right) !\right)=4 u_{i}-1+v\left(\left(4 u_{i}-1\right) !\right)=4 u_{i}-1+2 u_{i}-1+v\left(\left(2 u_{i}-1\right) !\right) \geq 4 u_{i} \geq e_{i}+1$, which verifies $(7)$.

Finally assume $\alpha=2$ and $i=3$. Then $v\left(\left(4 u_{i}-2\right) !\right)=2 u_{i}-1+v\left(\left(2 u_{i}-1\right) !\right)=$ $2 u_{i}-1+u_{i}-1+v\left(\left(u_{i}-1\right) !\right) \geq 3 u_{i} \geq 2 u_{i}+v\left(u_{i} !\right)+1$ if $u_{i} \geq 5$, while if $u_{i}=3$, then $v\left(\left(4 u_{i}-2\right) !\right)=v(10 !)=8=e_{i}+1$. So $(7)$ is true in this case.

Since we noted above that (7) holds whenever $r \geq 3$, we have established (7) for all but cases (i)-(v). If $u_{2}=1$ and $u_{3}>0$, then $(2+1) u_{2}+(3+1) u_{3}-2=4 u_{3}+1$ and $v\left(\left(4 u_{3}\right) !\right)=2 u_{3}+u_{3}+v\left(\left(u_{3}\right) !\right) \geq e_{2}+e_{3}+1$. Since $v\left(\left(\sum a_{j}\right) !\right) \geq \sum v\left(a_{j} !\right)$, we then deduce $v\left(\tau_{u}\right)>0$. Similarly if a term $\tau_{u}$ is different from the exceptional cases in (i) $-(\mathrm{v})$, then by $(7)$ we have $v\left(\tau_{u}\right)>0$. Thus we have only to consider the exceptional cases.

For case (i), we have

$$
\tau_{u}=(-1)^{n-1} \frac{(2 n-2) !}{2^{n} n !}=(-1)^{n-1} \frac{1}{2 n} \frac{(2 n-2) !}{2^{n-1}(n-1) !}=(-1)^{n-1} \frac{(2 n-3) ! !}{2 n} .
$$

Hence $v\left(\tau_{u}\right)=-(1+v(n))$.

For case (ii), we have

$$
\tau_{u}=(-1)^{n-1} \frac{(2 n-4) !}{2^{n-3}(n-3) ! 2^{2}}=(-1)^{n-1} \frac{(n-2)(2 n-5) ! !}{2} .
$$

Thus $v\left(\tau_{u}\right)=-1$ if $n$ is odd, $v\left(\tau_{u}\right)=0$ if $4 \mid n$, and $v\left(\tau_{u}\right)>0$ if $n \equiv 2(\bmod 4)$.

For case (iii), we have

$$
\tau_{u}=(-1)^{n-1} \frac{(2 n-6) !}{2^{n-6}(n-6) ! 2^{5}}=(-1)^{n-1} \frac{(n-3)(n-4)(n-5)(2 n-7) ! !}{2^{2}} .
$$

Therefore $v\left(\tau_{u}\right)>0$ if $n$ is odd or $n \equiv 4(\bmod 8)$, while if $n \equiv 2(\bmod 4)$, then $v\left(\tau_{u}\right)=-1$, and if $8 \mid n$, then $v\left(\tau_{u}\right)=0$.

For case (iv), we have

$$
\tau_{u}=(-1)^{n-1} \frac{(2 n-10) !}{2^{n-12}(n-12) ! 2^{11}}=(-1)^{n-1} \frac{(2 n-11) ! ! \prod_{i=5}^{11}(n-i)}{2^{4}} .
$$

Thus if $n \equiv 4(\bmod 8)$, then $v\left(\tau_{u}\right)=0$, and otherwise $v\left(\tau_{u}\right)>0$.

For case (v), we have

$$
\tau_{u}=(-1)^{n} \frac{(2 n-3) !}{2^{n-2}(n-2) ! 3}=(-1)^{n} \frac{(2 n-3) ! !}{3} .
$$

Therefore $v\left(\tau_{u}\right)=0$. This completes the proof of Theorem 2.3. 


\section{A simple proof of Clarke's universal von Staudt theorem}

In this section, we provide a simple proof to Clarke's universal von Staudt theorem. In order to state Clarke's congruence, we recall the defined numerical function $z(p, n)$ introduced in [4] which will also be used in the statements of our universal Kummer congruence in the next section. Let $p$ be a prime and $n$ a natural number divisible by $p-1$. So we may let $n=p^{N}(p-1) k$, where $p$ does not divide $k$. Then $z(p, n)$ is determined modulo $p^{N+1}$ as follows: If $p=2$ and $N=1$, then $z(2, n) \equiv-k$ $(\bmod 4)$, otherwise $z(p, n)$ is the multiplicative inverse of $k$ modulo $p^{N+1}$ so that $k z(p, n) \equiv 1\left(\bmod p^{N+1}\right)$. We first need a preliminary number-theoretic result from [4.

Lemma 3.1 (4, Proposition 2]). If $p$ is an odd prime and $a$ is divisible by $p^{N}$ with $N \geq 0$, then $\frac{(p a) !}{p^{a} a !} \equiv(-1)^{a}\left(\bmod p^{N+1}\right)$. If a is odd, then $\frac{(2 a) !}{2^{a} a !}$ is odd. If $a \equiv 2(\bmod 4)$, then $\frac{(2 a) !}{2^{a} a !} \equiv-1(\bmod 4)$. If a is divisible by $2^{N}$ with $N \geq 2$, then $\frac{(2 a) !}{2^{a} a !} \equiv 1\left(\bmod 2^{N+1}\right)$.

The above result has recently been strengthened by Clarke and Jones [5]. Using a different method from [5], we will strengthen the assertions for $p=2$ in the next section.

Theorem 3.1 ([4, Theorem 5]). If $n$ is divisible by 4 , then we have

$$
\frac{\hat{B_{n}}}{n} \equiv \sum_{\substack{n=s(p-1) \\ p \text { prime }}} \frac{z(p, n)}{p^{1+v(n)}} c_{p-1}^{s} \quad\left(\bmod \mathbf{Z}\left[c_{1}, c_{2}, \ldots\right]\right) .
$$

If $n$ is congruent to $2 \bmod 4$ and greater than 2 , then we have

$$
\frac{\hat{B_{n}}}{n} \equiv \frac{c_{1}^{n-6} c_{3}^{2}}{2}+\sum_{\substack{n=s(p-1) \\ p \text { prime }}} \frac{z(p, n)}{p^{1+v(n)}} c_{p-1}^{s} \quad\left(\bmod \mathbf{Z}\left[c_{1}, c_{2}, \ldots\right]\right) .
$$

If $n$ is odd and greater than 1, then we have

$$
\frac{\hat{B_{n}}}{n} \equiv \frac{c_{1}^{n}+c_{1}^{n-3} c_{3}}{2} \quad\left(\bmod \mathbf{Z}\left[c_{1}, c_{2}, \ldots\right]\right) .
$$

Proof. By (2), to prove Theorem 3.1, it is sufficient to show that each of the following is true:

(i) If $p$ is prime and $n=s(p-1)$ and $u_{p-1}=\frac{n}{p-1}=s$, then $\tau_{u} \equiv \frac{z(p, n)}{p^{1+v(n)}}$ $(\bmod \mathbf{Z})$.

(ii) If $n \equiv 2(\bmod 4)$ and $n>2$ and $u_{1}=n-6, u_{3}=2$, then $\tau_{u} \equiv \frac{1}{2}(\bmod \mathbf{Z})$.

(iii) If $n$ is odd and $n>1$ and $u_{1}=n-3, u_{3}=1$, then $\tau_{u} \equiv \frac{1}{2}(\bmod \mathbf{Z})$.

(iv) For every other monomial, $\tau_{u}$ is an integer.

If $p$ is odd and $v(n)=N$, or $p=2$ and $N>2$, then (i) is equivalent to $n p \tau_{u} \equiv p-1\left(\bmod p^{N+1}\right)$. But $n p \tau_{u}=\frac{(-1)^{s-1}(s p) !(p-1)}{(s p-1) p^{s} s !}$, so that Lemma 3.1 gives the required result.

If $p=2$ and $N=1$, then (i) is also equivalent to $n p \tau_{u} \equiv p-1\left(\bmod p^{N+1}\right)$, namely $\frac{(-1)^{s-1}(s p) !(p-1)}{(s p-1) p^{s} s !} \equiv p-1\left(\bmod p^{N+1}\right)$, i.e., $4 k \tau_{u}=\frac{(-1)^{2 k-1}(4 k-2) ! 4 k}{2^{2 k}(2 k) !} \equiv 1$ $(\bmod 4)$. But by Lemma 3.1, the latter is clearly true. Thus part (i) is proved. 
Parts (ii) and (iii) follow from Theorem 2.3. Part (iv) follows from the local estimates given in Theorem 2.1, or in Proposition 3.2 of [3], which are independent of the assumptions $(p-1) \nmid n$ or $(p-1) \mid n$. The proof of Theorem 3.1 is complete.

\section{Universal Kummer CONGRUEnCES}

Adelberg [2] discussed the universal Kummer congruences modulo $p$ for the divided universal Bernoulli numbers when $(p-1) \nmid n$. In this section, we establish the following universal Kummer congruences modulo $p$ for the case $(p-1) \mid n$, which is a new result.

If $(p-1) u_{p-1}=n$, then we define $z(p, n) \equiv p^{1+v(n)} \tau_{u}\left(\bmod p^{2+v(n)}\right)$. Equivalently, if $n=s(p-1)$ with $s=k p^{N}$ and $p \nmid k$, then we define $z(p, n)\left(\bmod p^{N+2}\right)$ to be such that

$$
z(p, n) \equiv(-1)^{s-1} \frac{(s p-2) ! p^{N+1}}{s ! p^{s}} \equiv(-1)^{s} \frac{(s p) !}{s ! p^{s} k\left(1-k p^{N+1}\right)} \quad\left(\bmod p^{N+2}\right),
$$

which extends the mod $p^{N+1}$ definition. We first give the universal Kummer congruences modulo an odd prime $p$.

Theorem 4.1. Let $p$ be an odd prime and let $n=s(p-1)$. Then the following hold.

(i) If $p \geq 5$, then

$\frac{\hat{B}_{n}}{n} \equiv \frac{z(p, n)}{p^{1+v(n)}} c_{p-1}^{s}+\left(2 c_{\frac{p-1}{2}}^{2}-\sum_{i=1}^{(p-3) / 2} \frac{1}{i(i+1)} c_{i} c_{p-1-i}\right) c_{p-1}^{s-1}\left(\bmod p \mathbf{Z}_{p}\left[c_{1}, \ldots, c_{n}\right]\right)$.

(ii) If $p=3$ and $n \geq 8$, then

$$
\begin{aligned}
& \frac{\hat{B}_{n}}{n} \equiv \frac{z(3, n)}{3^{1+v(n)}} c_{2}^{s}+2 c_{1}^{2} c_{2}^{s-1}+s(s-2) c_{2}^{\frac{n-8}{2}} c_{8} \quad\left(\bmod 3 \mathbf{Z}_{3}\left[c_{1}, \ldots, c_{n}\right]\right) . \\
& \text { If } p=3 \text { and } n<8, \text { delete the } c_{8} \text { term in the congruence. }
\end{aligned}
$$

Proof. By Theorem 2.2, we have only to consider the term $\tau_{u}$ when $u_{p-1}=s$ and the exceptional terms of Theorem 2.1.

If $u_{p-1}=s$, then $\frac{z(p, n)}{p^{1+v(n)}} \equiv \tau_{u}(\bmod p)$ by definition.

For the exceptional case where $u_{p-1}=s-1$ and $u_{\frac{p-1}{2}}=2$, we have

$$
\tau_{u}=(-1)^{s} \frac{(s p-1) !}{(s-1) ! 2 ! p^{s-1}\left(\frac{p+1}{2}\right)^{2}} \equiv 2 \quad(\bmod p) .
$$

For the exceptional case where $u_{p-1}=s-1$ and $u_{i}=u_{p-1-i}=1$, we have

$$
\tau_{u}=(-1)^{s} \frac{(s p-1) !}{(s-1) ! p^{s-1}(i+1)(p-1-i+1)} \equiv-\frac{1}{i(i+1)} \quad(\bmod p) .
$$

Finally if $p=3$ and $u_{2}=s-4, u_{8}=1$, then

$$
\begin{aligned}
\tau_{u} & =(-1)^{s} \frac{(3 s-5) !}{3^{s-4}(s-4) ! 3^{2}} \\
& \equiv(s-2)(s-3)(3 s-5)(3 s-7)(3 s-8)(3 s-10)(3 s-11) \\
& \equiv s(s-2) \quad(\bmod 3) .
\end{aligned}
$$

Thus if $s \equiv 1(\bmod 3)$, then $\tau_{u} \equiv 2(\bmod 3)$. So Theorem 4.1 is proved.

To illustrate Theorem 4.1, we give an example as follows. 
Example 4.1. By (2) and some computations we obtain the following congruences:

$$
\begin{aligned}
& \frac{\hat{B}_{4}}{4} \equiv 2 c_{1} c_{3}-\frac{4}{3} c_{2}^{2}+\frac{6}{5} c_{4} \quad(\bmod 5), \\
& \frac{\hat{B}_{8}}{8} \equiv 2 c_{1} c_{3} c_{4}+2 c_{2}^{2} c_{4}-\frac{7}{5} c_{4}^{2} \quad(\bmod 5), \\
& \frac{\hat{B}_{12}}{12} \equiv 2 c_{1} c_{3} c_{4}^{2}+2 c_{2}^{2} c_{4}^{2}-\frac{3}{5} c_{4}^{3} \quad(\bmod 5), \\
& \frac{\hat{B}_{16}}{16} \equiv 2 c_{1} c_{3} c_{4}^{3}+2 c_{2}^{2} c_{4}^{3}-\frac{1}{5} c_{4}^{4} \quad(\bmod 5), \\
& \frac{\hat{B}_{6}}{6} \equiv 3 c_{1} c_{5}+c_{2} c_{4}+2 c_{3}^{2}+\frac{22}{7} c_{6} \quad(\bmod 7), \\
& \frac{\hat{B}_{12}}{12} \equiv 3 c_{1} c_{5} c_{6}+c_{2} c_{4} c_{6}+2 c_{3}^{2} c_{6}-\frac{3}{7} c_{6}^{2} \quad(\bmod 7), \\
& \frac{\hat{B}_{18}}{18} \equiv 3 c_{1} c_{5} c_{6}^{2}+c_{2} c_{4} c_{6}^{2}+2 c_{3}^{2} c_{6}^{2}+\frac{5}{7} c_{6}^{3} \quad(\bmod 7), \\
& \frac{\hat{B}_{8}}{8} \equiv 2 c_{1}^{2} c_{2}^{3}+2 c_{8}-\frac{2}{3} c_{2}^{4} \quad(\bmod 3), \\
& \frac{\hat{B}_{14}}{14} \equiv 2 c_{1}^{2} c_{2}^{6}+2 c_{2}^{3} c_{8}+\frac{4}{3} c_{2}^{7} \quad(\bmod 3), \\
& \frac{\hat{B}_{20}}{20} \equiv 2 c_{1}^{2} c_{2}^{9}+2 c_{2}^{6} c_{8}+\frac{1}{3} c_{2}^{10} \quad(\bmod 3), \\
& \frac{\hat{B}_{2}}{2} \equiv-\frac{1}{4} c_{1}^{2}+\frac{1}{3} c_{2} \quad(\bmod 3), \\
& \frac{\hat{B}_{4}}{4} \equiv 2 c_{1}^{2} c_{2}^{1}-\frac{4}{3} c_{2}^{2} \quad(\bmod 3), \\
& \frac{\hat{B}_{6}}{6} \equiv 2 c_{1}^{2} c_{2}^{2}+\frac{10}{9} c_{2}^{3} \quad(\bmod 3) .
\end{aligned}
$$

where we use $\bmod p$ to abbreviate $\bmod p \mathbf{Z}_{p}\left[c_{1}, c_{2}, \ldots\right]$ for a prime $p$.

Lemma 4.1. (i) If $k$ is odd, then $(2 k-1) ! ! \equiv(-1)^{\frac{k-1}{2}}(\bmod 4)$.

(ii) If $k \geq 1$, then $(4 k-3) ! ! \equiv(-1)^{k-1}(\bmod 16)$.

(iii) If $k \geq 1$ and $N \geq 3$, then $\left(k 2^{N}-3\right) ! ! \equiv-1\left(\bmod 2^{N+1}\right)$.

Proof. The proofs of parts (i) and (ii) are standard arguments using inductions on $k$ and we here omit the details of the proofs.

For part (iii), the base case $k=1$ is non-trivial. Let $N \geq 3$ and $k=1$. Let $S=\left\{3,5, \ldots, 2^{N}-3\right\}$. Then $|S|=2^{N-1}-2$ is congruent to 2 modulo 4 . If $a \in S$, there is a unique $b \in S$ satisfying $a b \equiv 1$ or $a b \equiv-1\left(\bmod 2^{N+1}\right)$ and $b \neq a(b \neq a$ since $S$ contains no elements $\bmod 2^{N+1}$ of order 2 and $\left.a^{2} \equiv 1(\bmod 4)\right)$. If $1 / a \notin S$, then $\frac{1}{a} \in-S$ and $b=-\frac{1}{a} \in S$, where the operations are taken and $\bmod 2^{N+1}$.

Partition the 2-element subsets $\{a, b\}$ where $b=-1 / a$ into pairs by grouping $\{a, b\}$ and $\left\{2^{N}-a, 2^{N}-b\right\}$. These two sets are distinct except when $\{a, b\}=$ $\left\{2^{N-1}-1,2^{N-1}+1\right\}$ since $a^{2} \equiv 1\left(\bmod 2^{N}\right)$ if and only if $a=2^{N-1} \pm 1$. Thus the number of these sets is odd, and $\prod_{x \in S} x \equiv-1\left(\bmod 2^{N+1}\right)$. So part (iii) is true for the case $k=1$. 
Now consider the general case. We may let $k \geq 2$. Since $|S|$ is even and the elements in $S$ are odd, we deduce that for $1 \leq l \leq k-1$,

$$
\prod_{x \in S}\left(x+l 2^{N}\right) \equiv \prod_{x \in S} x \quad\left(\bmod 2^{N+1}\right) .
$$

Since $\left(j 2^{N}+1\right)\left(j 2^{N}-1\right) \equiv-1\left(\bmod 2^{N+1}\right)$ for $1 \leq j \leq k-1$, we have

$$
\begin{aligned}
\left(k 2^{N}-3\right) ! ! & =\prod_{l=0}^{k-1} \prod_{x \in S}\left(x+l 2^{N}\right) \prod_{j=1}^{k-1}\left(j 2^{N}+1\right)\left(j 2^{N}-1\right) \\
& \equiv(-1)^{k}(-1)^{k-1}=-1 \quad\left(\bmod 2^{N+1}\right) .
\end{aligned}
$$

Thus part (iii) is proved, which ends the proof of Lemma 4.1.

Since $\frac{(2 a) !}{2^{a} a !}=(2 a-1) !$ !, we can now strengthen Lemma 3.1 for $p=2$ as follows.

Corollary 4.1. (i) If a is odd, then $\frac{(2 a) !}{2^{a} a !} \equiv(-1)^{\frac{a-1}{2}}(\bmod 4)$.

(ii) If $a=2 k$ with $k$ odd, then $\frac{(2 a) !}{2^{a} a !} \equiv 4 k-1(\bmod 16)$, and consequently $\frac{(2 a) !}{2^{a} a !} \equiv 3$ $(\bmod 8)$.

(iii) If $a=k 2^{N}$ with $k$ odd and $N \geq 2$, then $\frac{(2 a) !}{2^{a} a !} \equiv 1-2^{N+1}\left(\bmod 2^{N+2}\right)$.

We can use the preceding congruences to prove explicit congruences for $z(2, n)$ $\left(\bmod 2^{N+2}\right)$.

Lemma 4.2. Let $n=k 2^{N}$ with $k$ odd. Then each of the following is true.

(i) If $N=0$ (i.e., $n$ is odd), then $z(2, n) \equiv 1(\bmod 4)$.

(ii) If $N=1$ (i.e., $n \equiv 2(\bmod 4))$, then $z(2, n) \equiv-\frac{1}{k}(\bmod 8)$.

(iii) If $N>1$ (i.e., $4 \mid n)$, then $z(2, n) \equiv \frac{1}{k}\left(\bmod 2^{N+2}\right)$.

Proof. First we have

$$
\begin{aligned}
z(2, n) & \equiv \frac{2^{N+1}(-1)^{n-1}(2 n-2) !}{2^{n} n !}=\frac{(-1)^{n-1}(2 n-2) !}{k 2^{n-1}(n-1) !} \\
& =\frac{(-1)^{n-1}(2 n-3) ! !}{k} \quad\left(\bmod 2^{N+2}\right) .
\end{aligned}
$$

So for case (i), noting that $2 k-1 \equiv 1(\bmod 4)$, Lemma $4.1(\mathrm{i})$ gives us

$$
z(2, n) \equiv(-1)^{k-1}(-1)^{\frac{k-1}{2}} \cdot \frac{1}{k} \equiv 1 \quad(\bmod 4) .
$$

For case (ii), by Lemma 4.1(ii) we get

$$
z(2, n) \equiv \frac{(-1)^{n-1}}{k}(4 k-3) ! ! \equiv-\frac{1}{k}(-1)^{k-1} \equiv-\frac{1}{k} \quad(\bmod 8) .
$$

For case (iii), by Lemma 4.1(iii) we get

$$
z(2, n) \equiv \frac{(-1)^{n-1}}{k}\left(k 2^{N+1}-3\right) ! ! \equiv\left(-\frac{1}{k}\right)(-1) \equiv \frac{1}{k} \quad\left(\bmod 2^{N+2}\right)
$$

as desired. So Lemma 4.2 is proved.

We can now prove the following explicit mod 2 universal Kummer congruences.

Theorem 4.2. Let $p=2$ and $n=k 2^{N}$ with $k$ odd. Then we have the following.

(i) If $n \geq 3$ and $N=0$ (i.e., $n$ is odd), then

$$
\frac{\hat{B}_{n}}{n} \equiv \frac{1}{2}\left(c_{1}^{n}+c_{1}^{n-3} c_{3}\right)+c_{1}^{n-2} c_{2} \quad\left(\bmod 2 \mathbf{Z}_{2}\left[c_{1}, \ldots, c_{n}\right]\right) .
$$


(ii) If $n \geq 6$ and $N=1$ (i.e., $n \equiv 2(\bmod 4))$, then

$$
\frac{\hat{B}_{n}}{n} \equiv-\frac{1}{4 k} c_{1}^{n}+\frac{(-1)^{\frac{n-2}{4}}}{2} c_{1}^{n-6} c_{3}^{2}+c_{1}^{n-2} c_{2}\left(\bmod 2 \mathbf{Z}_{2}\left[c_{1}, \ldots, c_{n}\right]\right) \text {. }
$$

(iii) If $n \geq 12$ and $N>1$ (i.e., $4 \mid n)$, then

$$
\begin{gathered}
\frac{\hat{B}_{n}}{n} \equiv \frac{1}{2^{N+1} k} c_{1}^{n}+c_{1}^{n-3} c_{3}+\left(\frac{n}{4}-1\right) c_{1}^{n-6} c_{3}^{2}+\frac{n}{4} c_{1}^{n-12} c_{3}^{4}+c_{1}^{n-2} c_{2} \\
\left(\bmod 2 \mathbf{Z}_{2}\left[c_{1}, \ldots, c_{n}\right]\right) .
\end{gathered}
$$

If $n=1$ in $(i), 2$ in $(i i)$, or 4 or 8 in (iii), delete from the congruence all terms containing $c_{1}^{j}$ with $j<0$.

Proof. Since $\frac{z(2, n)}{2^{N+1}} \equiv \tau_{u}(\bmod 2)$ if $u_{1}=n$, by Lemmas 4.1 and 4.2 and Theorem 2.3 , it is sufficient to verify the congruences in the two cases where $u_{1}<n$ and $v\left(\tau_{u}\right)=-1$, namely

(I) $n$ is odd and $u_{1}=n-3, u_{3}=1$, and

(II) $n \equiv 2(\bmod 4)$ and $u_{1}=n-6, u_{3}=2$.

For case (I), we have seen in the proof of Theorem 2.3 that

$$
2 \tau_{u}=(-1)^{n-1}(n-2)(2 n-5) ! !,
$$

so by Lemma 4.1 we have for $n$ odd, $2 \tau_{u} \equiv(n-2)(-1)^{\frac{n-3}{2}} \equiv 1(\bmod 4)$.

Finally, for the exceptional case (II), we proved that

$$
2 \tau_{u}=(-1)^{n-1}(n-3)(n-4)(n-5)(2 n-7) ! ! / 2,
$$

so if $n=2 k$ with $k$ odd, then $2 \tau_{u}=-(2 k-3)(k-2)(2 k-5)(4 k-7)$ !!. Therefore by Lemma 4.1(ii) we have

$$
2 \tau_{u} \equiv-(-1)(k-2)(-3)(-1)^{k} \equiv 2-k \equiv(-1)^{\frac{k-1}{2}} \quad(\bmod 4) .
$$

The proof of Theorem 4.2 is complete.

Example 4.2. We have the following congruences modulo $2 \mathbf{Z}_{2}\left[c_{1}, c_{2}, \ldots\right]$ :

$$
\begin{aligned}
& \frac{\hat{B}_{4}}{4} \equiv \frac{1}{8} c_{1}^{4}+c_{1}^{2} c_{2}+c_{1} c_{3}, \quad \frac{\hat{B}_{5}}{5} \equiv \frac{1}{2} c_{1}^{5}+c_{1}^{3} c_{2}+\frac{1}{2} c_{1}^{2} c_{3}, \\
& \frac{\hat{B}_{6}}{6} \equiv \frac{5}{4} c_{1}^{6}+c_{1}^{4} c_{2}-\frac{1}{2} c_{3}^{2}, \quad \frac{\hat{B}_{7}}{7} \equiv \frac{1}{2} c_{1}^{7}+c_{1}^{5} c_{2}+\frac{1}{2} c_{1}^{4} c_{3}, \\
& \frac{\hat{B}_{8}}{8} \equiv \frac{1}{16} c_{1}^{8}+c_{1}^{6} c_{2}+c_{1}^{5} c_{3}+c_{1}^{2} c_{3}^{2}, \quad \frac{\hat{B}_{9}}{9} \equiv \frac{1}{2}\left(c_{1}^{9}+c_{1}^{6} c_{3}\right)+c_{1}^{7} c_{2}, \\
& \frac{\hat{B}_{10}}{10} \equiv-\frac{1}{20} c_{1}^{10}+\frac{1}{2} c_{1}^{4} c_{3}^{2}+c_{1}^{8} c_{2}, \quad \frac{\hat{B}_{12}}{12} \equiv \frac{1}{24} c_{1}^{12}+c_{1}^{9} c_{3}+c_{3}^{4}+c_{1}^{10} c_{2} .
\end{aligned}
$$

Finally we give some non-trivial applications of the $\bmod p$ universal Kummer congruences when $(p-1) \mid n$ to the classical Bernoulli numbers $B_{n}$ (where $c_{i}=(-1)^{i}$ and $\left.c^{u}=(-1)^{w(u)}\right)$. If $z(p, n)$ is defined as above, using the telescoping property for $\sum \frac{1}{i(i+1)}=\sum\left(\frac{1}{i}-\frac{1}{i+1}\right)$, we then obtain the following result.

Corollary 4.2. Let $n$ be a positive integer and $p$ a prime such that $(p-1) \mid n$. Then we have the following.

(i) If $p \geq 5$, then

$$
\frac{B_{n}}{n} \equiv \frac{z(p, n)}{p^{1+v(n)}}-1 \quad(\bmod p) .
$$


(ii) If $p=3$, then the congruence is the same as (8), except that if $n \geq 8$ and $n \equiv 2(\bmod 6)$, then

$$
\frac{B_{n}}{n} \equiv \frac{z(3, n)}{3^{1+v(n)}}+1 \quad(\bmod 3)
$$

(iii) If $p=2$, then the congruences are trivial if $n$ is odd. If $n \geq 8$ and $4 \mid n$, then we have the same congruence (8), namely

$$
\frac{B_{n}}{n} \equiv \frac{1}{2 n}-1 \quad(\bmod 2)
$$

If $n \geq 6$ and $n \equiv 2(\bmod 4)$, then we have

$$
\frac{B_{n}}{n} \equiv-\frac{1}{2 n}-1+\frac{(-1)^{\frac{n-2}{4}}}{2} \quad(\bmod 2) .
$$

[1] A. Adelberg, Universal higher order Bernoulli numbers and Kummer and related congruences, J. Number Theory 84 (1) (2000), 119-135. MR1782265 (2001g:11018)

[2] A. Adelberg, Kummer congruences for universal Bernoulli numbers and related congruences for poly-Bernoulli numbers, Int. Math. J. 1 (1) (2002), 53-63. MR1825492 (2002d:11023)

[3] A. Adelberg, Universal Kummer congruences mod prime powers, J. Number Theory 109 (2004), 362-378. MR2106486 (2005j:11018)

[4] F. Clarke, The universal von Staudt theorem, Trans. Amer. Math. Soc. 315 (1989), 591-603. MR.986687 (90a:11026)

[5] F. Clarke and C. Jones, A congruence for factorials, Bull. London Math. Soc. 36 (2004), 553-558. MR2069019(2005b:11020)

[6] I. Dibag, An analogue of the von Staudt-Clausen theorem, J. Algebra 87 (1984), 332-341. MR739937 (85j:11028)

[7] S. Hong, Notes on Glaisher's congruences, Chinese Ann. Math. 21B (2000), 33-38. MR.1762270 (2001e:11007)

[8] A. Hurwitz, Uber die Entwicklungskoeffizienten der lemniskatischen Funktionen, Math. Ann. 51 (1899), 196-226.

[9] N.M. Katz, The congruences of Clausen-von Staudt and Kummer for Bernoulli-Hurwitz numbers, Math. Ann. 216 (1975), 1-4. MR0387293 (52:8136)

[10] M.R. Murty, Introduction to p-adic analytic number theory, Studies in Advanced Mathematics, vol. 27, American Math. Soc., Providence, RI, 2002. MR1913413 (2003c:11151)

[11] N. Ray, Extensions of umbral calculus I: Penumbral coalgebras and generalised Bernoulli numbers, Adv. Math. 61 (1986), 41-100. MR847728 (88b:05019)

[12] W. Ren, S. Hong, and X. Zhou, A generalization of Wilson's theorem, J. Sichuan Univ. Nat. Sci. Ed. 43 (2006), 517-519. MR.2241947(2007a:11006)

[13] L. Washington, p-adic L-functions and sums of powers, J. Number Theory 69 (1998), 50-61. MR1611077 (99a:11134)

Department of Mathematics, Grinnell College, Grinnell, Iowa 50112-0806

E-mail address: adelbe@math.grinnell.edu CHINA

Mathematical College, Sichuan University, Chengdu 610064, People's Republic of

$$
\text { E-mail address: s-f.hong@tom.com }
$$

E-mail address: hongsf02@yahoo.com

Mathematical College, Sichuan University, Chengdu 610064, People's Republic of China, and Department of Mathematics, Dezhou University, Dezhou 253023, People's Republic of China

E-mail address: renwenli80@163.com 